\title{
Distributed Average Consensus in Sensor Networks with Quantized Inter-Sensor Communication
}

\author{
Soummya Kar and José M. F. Moura \\ Department of Electrical and Computer Engineering \\ Carnegie Mellon University, Pittsburgh, PA 15213 USA \\ Email: soummyak@andrew.cmu.edu,moura@ece.cmu.edu
}

\begin{abstract}
The paper studies distributed average consensus in sensor networks, when the sensors exchange quantized data at each time step. We show that randomizing the exchanged sensor data by adding a controlled amount of dither results in almost sure (a.s.) convergence of the protocol, if the network is connected. We explicitly characterize the mean-squared error (with respect to the desired consensus average) and show that, by tuning certain parameters associated with the protocol, the mean-squared error can be made arbitrarily small. We study the trade-offs between the rate of convergence and the resulting mean-squared error. The sensor network topology plays an important role in determining the convergence rate of the algorithm. Our approach, based on the convergence of controlled Markov processes, is very generic and can be applied to many other situations of imperfect communication. Finally, we present numerical studies, which verify our theoretical results.
\end{abstract}

Index Terms-Distributed Consensus, Quantized Information Exchange, Topology, Laplacian, Randomized Algorithm.

\section{INTRODUCTION}

Distributed average consensus computes the global average of sensor data in a distributed fashion in sensor networks, using only local inter-sensor communication (see, e.g., [1].) It finds applications in many practical problems in distributed sensor networking, including distributed detection, estimation, swarm aggregation, flocking. In most cases, these decentralized networking applications operate under severe resource constraints, both in terms of computation and communication. In particular, sensors in a wireless networked environment communicate by exchanging quantized rather than analog data, where the bandwidth or data rate allocated to a particular inter-sensor communication channel is directly related to the number of bits used by the quantizer. In this paper, we provide an algorithm, QC, for average consensus with quantized intersensor information exchange and analyze its performance and convergence properties.

We highlight several key features of our algorithm. First, we note that, with no randomization, the quantization errors will, in general, accumulate and lead to divergence and unboundedness of the sensor states. Thus, deliberately, we randomize our algorithm by adding a controlled amount of

This work was partially supported by the DARPA DSO Advanced Computing and Mathematics Program Integrated Sensing and Processing (ISP) Initiative under ARO grant \# DAAD 19-02-1-0180, by NSF under grants \# ECS-0225449 and \# CNS-0428404, by the Office of Naval Research under MURI N000140710747, and by an IBM Faculty Award. statistical dither to the sensor data before quantization. This results in good statistical properties of the quantization error sequence, which are exploited in the algorithm. Another key feature is that the algorithm uses a properly scaled sequence of decreasing link weights, which keep the error resulting from randomization and quantization uniformly bounded at each step (to be explained in more detail in the paper.) We use results from the theory of controlled Markov processes that show that, for a connected network, our algorithm leads to almost sure (a.s.) convergence of the sensor states to the same finite random variable. In other words, the sensors asymptotically reach consensus and we explicitly characterize the mean-squared error (m.s.e.) between the desired average and the converged sensor states. The m.s.e. can be made arbitrarily small by rescaling the sequence of link weights associated with the algorithm. However, improving the accuracy of the final estimate in this way, slows down the convergence rate of the algorithm and we find an interesting trade-off between convergence rate and m.s.e. Finally, we note that our approach is very generic and can be extended with similar results to other situations of imperfect communication, like random link failure, additive noise, etc. Due to lack of space, we will mainly highlight the key steps in the derivation of these results. Detailed derivation and analysis can be found in [2].

The distributed consensus problem with quantized transmission has been studied recently in [3], [4]. The algorithm in [3] is restricted to integer-valued initial sensor states, where at each iteration the sensors exchange integer-valued data. It is shown there that the sensor states are asymptotically close (in the appropriate sense defined there) to the desired average, but may not reach absolute consensus. In [4], a randomized algorithm is considered, and it is shown that the expected sensor states converge to the desired average, but no analytical bounds on the mean-squared error are presented. In contrast, the QC algorithm considered in this paper, leads to a.s. consensus of the sensor states to a finite random variable, in addition to the fact that the expected sensor states converge to the desired average. We explicitly characterize the resulting m.s.e. and show that, by tuning certain parameters of our algorithm, the m.s.e. can be made arbitrarily small, though at a cost of convergence rate.

We comment briefly on the organization of the overall paper. Section II sets up the problem and reviews preliminary concepts. The proof of a.s. convergence of the randomized algorithm is in Section III. We study the trade-offs between 
the m.s.e and the convergence rate in Section IV. Section V presents a numerical study. Finally, Section VI concludes the paper and comments on the generalizations of our approach.

\section{Problem Formulation AND PRELIMINARIES}

We model the sensor network by a graph $G=(V, E)$, where $V$ represents the set of $N$ nodes in the network and $E$ is the set of $M$ edges or communication links among the sensors. For clarity, we assume that the network topology remains unchanged throughout the process. However, our approach can handle randomly varying topologies, as commented in Section VI. We define the $N \times N$ adjacency matrix, $A$, as

$$
A_{n, l}= \begin{cases}1 & \text { if }(n, l) \in E \\ 0 & \text { otherwise }\end{cases}
$$

The graph Laplacian, $L$, is then defined as

$$
L=D-A
$$

where $D=\operatorname{diag}\left(d_{1} \cdots d_{N}\right)$ is the diagonal matrix of node degrees. ${ }^{1}$ The Laplacian $L$ is a symmetric positive-semidefinite matrix and hence its eigenvalues can be arranged as

$$
0=\lambda_{1}(L) \leq \lambda_{2}(L) \leq \cdots \leq \lambda_{N}(L(i))
$$

For a connected network $\lambda_{2}(L)>0$ (see [5].) Throughout the paper, we assume that the sensor network is connected.

Let $\mathbf{x}(0) \in \mathbb{R}^{N \times 1}$ be the vector of initial sensor states. Define the average, $r=(1 / N) \mathbf{1}^{T} \mathbf{x}(0)$, where $\mathbf{1}$ is the $N \times 1$ vector of ones. In distributed average consensus, starting from some initial state, $\mathbf{x}(0)$, the sensors compute the average $r$ iteratively, where at each iteration each sensor has access only to its neighboring states. For consensus with quantization, the sensors can exchange only quantized state information with their neighbors. In particular, we assume that each inter-sensor communication channel uses a uniform quantizer, whose input-output relation may be modeled by the quantizing function ${ }^{2}, q(\cdot): \mathbb{R} \rightarrow \mathcal{Q}$,

$$
q(y)=b \Delta, \quad\left(b-\frac{1}{2}\right) \Delta \leq y<\left(b+\frac{1}{2}\right) \Delta
$$

where $y \in \mathbb{R}$ is the channel input and $\Delta>0$ is the quantization step-size. We may write

$$
q(y)=y+e(y)
$$

where $e(y)$ is the quantization error, and we have

$$
-\frac{\Delta}{2} \leq e(y) \leq \frac{\Delta}{2}, \forall y
$$

We now present our randomized algorithm QC for quantized consensus.

QC Algorithm: Define the consensus subspace, $\mathcal{C}$, as

$$
\mathcal{C}=\left\{x \in \mathbb{R}^{N \times 1} \mid x=a \mathbf{1}, a \in \mathbb{R}\right\}
$$

\footnotetext{
${ }^{1}$ The neighborhood of a node $n$ in $G$ is defined as $\Omega_{n}=$ $\{l \in V:(n, l) \in E(i)\}$. The corresponding node degree, $d_{n}$ is the number of edges incident to it and is given by $d_{n}=\left|\Omega_{n}\right|$.

${ }^{2}$ In this case, the quantizer output takes values in the countable set, $\mathcal{Q}=$ $\{k \Delta \mid k \in \mathbb{Z}\}$. However, if the initial state $\mathbf{x}(0)$ is bounded, only finitelymany quantization steps will suffice (see [2].)
}

Consider the sequence $\left\{\nu_{n l}(i)\right\}_{i \geq 0,1 \leq n, l<N}$ of i.i.d. random variables uniformly distributed on $[-\Delta / 2, \Delta / 2)$. Let $\{\alpha(i)\}_{i \geq 0}$ be a real number sequence satisfying

$$
\alpha(i)>0 \quad \sum_{i \geq 0} \alpha(i)=\infty \quad \sum_{i \geq 0} \alpha^{2}(i)<\infty
$$

Denoting by $x_{n}(i)$ the state of the $n$-th sensor at iteration $i$, the QC algorithm consists of the following recursion:

$$
x_{n}(i+1)=\left(1-\alpha(i) d_{n}\right) x_{n}(i)+\alpha(i) \sum_{l \in \Omega_{n}} q\left(x_{l}(i)+\nu_{n l}(i)\right)
$$

with the initial sensor state vector $\mathbf{x}(0)$.

In Section III, we show that, under the QC algorithm, the state vector sequence, $\{\mathbf{x}(i)\}_{i \geq 0}$, converges a.s. to the consensus subspace $\mathcal{C}$. In other words, there exists a finite random variable, $\theta$, such that

$$
\mathbb{P}\left[\lim _{i \rightarrow \infty} \mathbf{x}(i)=\theta \mathbf{1}\right]=1
$$

The m.s.e, $\zeta$, is then given by

$$
\zeta=\mathbb{E}[\theta-r]^{2}
$$

and this is explicitly characterized in Section IV.

\section{Proof of Convergence of QC}

The recursive update in eqn. (9) is rewritten as

$$
\begin{aligned}
x_{n}(i+1)= & \left(1-\alpha(i) d_{n}\right) x_{n}(i)+\alpha(i) \sum_{l \in \Omega_{n}}\left(x_{l}(i)\right. \\
& \left.+\nu_{n l}(i)+\varepsilon_{n l}(i)\right)
\end{aligned}
$$

where $\varepsilon_{n l}(i)$ is the quantization error and given by

$$
\varepsilon_{n l}(i)=q\left(x_{l}(i)+\nu_{n l}(i)\right)-x_{l}(i)-\nu_{n l}(i)
$$

By construction, the i.i.d. set $\left\{\nu_{n l}(i)\right\}_{1 \leq n, l<N}$ is independent of $\mathbf{x}(i)$. Then, it can be shown from results in statistical quantization theory (see [6]) that the set $\left\{\varepsilon_{n l}(i)\right\}_{1 \leq n, l \leq N}$ consists of i.i.d. random variables uniformly distributed on $[-\Delta / 2, \Delta / 2)$ and independent of $\mathbf{x}(i)$. Introduce

$$
\Upsilon_{n}(i)=-\sum_{l \in \Omega_{n}} \nu_{n l}(i), \quad \Psi_{n}(i)=-\sum_{l \in \Omega_{n}} \varepsilon_{n l}(i)
$$

The update in eqn. (12) can be put in vector form as

$$
\mathbf{x}(i+1)=\mathbf{x}(i)-\alpha(i)[L \mathbf{x}(i)+\mathbf{\Upsilon}(i)+\mathbf{\Psi}(i)]
$$

where the random vectors $\boldsymbol{\Upsilon}(i)$ and $\boldsymbol{\Psi}(i)$ are independent of the state $\mathbf{x}(i)$ and have independent components.

We now give a result on the convergence of sample paths of Markov processes, which will be used later.

Theorem 1 Consider a Markov process, $\{\mathbf{x}(i)\}_{i \geq 0}$ on $\mathbb{R}^{N \times 1}$. Define the operator $\mathcal{L}$, which acts on non-negative functions $V(i, \mathbf{x}), i \geq 0, x \in \mathbb{R}^{N \times 1}$ by

$\mathcal{L} V(i, \mathbf{x})=\mathbb{E}[V(i+1, \mathbf{x}(i+1)) \mid \mathbf{x}(i)=\mathbf{x}]-V(i, \mathbf{x})$ a.s. 
Now suppose there exists a non-negative function $V(i, \mathbf{x}), \quad i \geq 0, x \in \mathbb{R}^{N \times 1}$ and a set $B \subset \mathbb{R}^{N \times 1}$ with the following properties:

1)

$$
\begin{gathered}
\inf _{i \geq 0, x \in V_{\epsilon}(B)} V(i, \mathbf{x})>0, \forall \epsilon>0 \\
V(i, \mathbf{x}) \equiv 0, \mathbf{x} \in B, \quad \lim _{\mathbf{x} \rightarrow B} \sup _{i \geq 0} V(i, \mathbf{x})=0
\end{gathered}
$$

where $V_{\epsilon}(B)=\left\{x \in \mathbb{R}^{N \times 1} \mid \inf _{y \in B} \rho(x, y) \geq \epsilon\right\}$. 2)

$$
\mathcal{L} V(i, \mathbf{x}) \leq g(i)(1+V(i, \mathbf{x}))-\alpha(i) \varphi(i, \mathbf{x})
$$

where $\varphi(i, \mathbf{x}), i \geq 0, \mathbf{x} \in \mathbb{R}^{N \times 1}$ is a non-negative function such that

$$
\inf _{i, \mathbf{x} \in V_{\epsilon}(B)} \varphi(i, \mathbf{x})>0, \forall \epsilon>0
$$

3)

$$
\alpha(i), g(i)>0, \quad \sum_{i \geq 0} \alpha(i)=\infty, \quad \sum_{i \geq 0} g(i)<\infty
$$

Then, the Markov process $\left\{\mathbf{x}_{i}\right\}_{i \geq 0}$ with arbitrary initial distribution converges a.s. to $\mathrm{B}$ as $i \rightarrow \infty$.

Proof: The proof is given in [2] and relies on [7]. We omit it here due to lack of space.

We are now in a position to give the main theorem on the a.s. convergence of the QC algorithm.

Theorem 2 Let $\mathbf{x}(0)$ be the initial sensor state vector and $r$ the corresponding average, to be computed. Consider the state vector sequence, $\{\mathbf{x}(i)\}_{i \geq 0}$, generated by the QC algorithm. Then there exists a finite random variable $\theta$, such that

$$
\mathbb{P}\left[\lim _{i \rightarrow \infty} \mathbf{x}(i)=\theta \mathbf{1}\right]=1
$$

Proof: We outline the key steps of the proof. Details can be found in [2]. It is clear that the state vector sequence $\{\mathbf{x}(i)\}_{i \geq 0}$ generated by the QC algorithm is a Markov process. Assuming the network is connected, it can be shown that this Markov process satisfies the conditions of Theorem 1 with

$$
V(i, \mathbf{x})=\mathbf{x}^{T} L \mathbf{x}, \quad B=\mathcal{C}
$$

where $L$ is the graph Laplacian and $\mathcal{C}$ is the consensus subspace, defined in eqn. (7). We then have, by Theorem 1, that $\mathbf{x}(i)$ converges a.s. to the set $\mathcal{C}$. This is equivalent to the fact that, asymptotically, the components of $\mathbf{x}(i)$ reach consensus and hence tend to their average value. In other words, we have

$$
\mathbb{P}\left[\lim _{i \rightarrow \infty}\left\|\mathbf{x}(i)-x_{\text {avg }}(i) \mathbf{1}\right\|=0\right]=1
$$

where, $x_{\mathrm{avg}}(i)=(1 / N) \mathbf{1}^{T} \mathbf{x}(i)$ is the average of the sensor states at time $i$, with $x_{\text {avg }}(0)=r$.

The proof will be completed if we can show that the random sequence, $\left\{x_{\text {avg }}(i)\right\}_{i \geq 0}$, converges a.s. to some finite random variable $\theta$. To this end, we note that the sequence $\left\{x_{\text {avg }}(i)\right\}_{i \geq 0}$ is given by the following recursion:

$$
x_{\mathrm{avg}}(i+1)=x_{\mathrm{avg}}(i)-\alpha(i)[\bar{\Upsilon}(i)+\bar{\Psi}(i)], \quad x_{\mathrm{avg}}(0)=r
$$

where $\bar{\Upsilon}(i)=(1 / N) \mathbf{1}^{T} \boldsymbol{\Upsilon}(i)$ and $\bar{\Psi}(i)=(1 / N) \mathbf{1}^{T} \boldsymbol{\Psi}(i)$. Clearly, $\bar{\Upsilon}(i)$ and $\bar{\Psi}(i)$ are independent of $\mathbf{x}(i)$. Also, it follows from the fact that the components of the sets $\left\{\nu_{n l}(i)\right\}_{1 \leq n, l \leq N}$ and $\left\{\varepsilon_{n l}(i)\right\}_{1 \leq n, l \leq N}$ are i.i.d. uniformly distributed on $[-\Delta / 2, \Delta / 2)$, that

$$
\mathbb{E}[\bar{\Upsilon}(i)]=\mathbb{E}[\bar{\Psi}(i)]=0, \forall i
$$

Also, we have

$$
\mathbb{E}\left[\bar{\Upsilon}^{2}(i)\right]=\mathbb{E}\left[\bar{\Psi}^{2}(i)\right]=\frac{M \Delta^{2}}{6 N^{2}}, \forall i
$$

where $M$ is the number of edges in the network. It can be shown that the sequence $\left\{x_{\text {avg }}(i)\right\}_{i \geq 0}$ is a martingale with respect to the filtration ${ }^{3}$

$$
\mathcal{F}_{i}=\sigma\left\{\mathbf{x}(0),\{\boldsymbol{\Upsilon}(j)\}_{0 \leq j<i},\{\boldsymbol{\Psi}(j)\}_{0 \leq j<i}\right\}
$$

We then have

$$
\begin{aligned}
\mathbb{E}\left[x_{\text {avg }}^{2}(i+1)\right]= & \mathbb{E}\left[x_{\text {avg }}(i)-\alpha(i)[\bar{\Upsilon}(i)+\bar{\Psi}(i)]\right]^{2} \\
= & \mathbb{E}\left[x_{\text {avg }}^{2}(i)\right]+\alpha^{2}(i)\left[\mathbb{E}\left[\bar{\Upsilon}^{2}(i)\right]\right. \\
& \left.+\mathbb{E}\left[\bar{\Psi}^{2}(i)\right]+2 \mathbb{E}[\bar{\Upsilon}(i) \bar{\Psi}(i)]\right] \\
\leq & \mathbb{E}\left[x_{\text {avg }}^{2}(i)\right]+\alpha^{2}(i)\left[\mathbb{E}\left[\bar{\Upsilon}^{2}(i)\right]+\mathbb{E}\left[\bar{\Psi}^{2}(i)\right]\right. \\
& \left.+2\left(\mathbb{E}\left[\bar{\Upsilon}^{2}(i)\right]\right)^{1 / 2}\left(\mathbb{E}\left[\bar{\Psi}^{2}(i)\right]\right)^{1 / 2}\right] \\
= & \mathbb{E}\left[x_{\text {avg }}^{2}(i)\right]+\frac{2 \alpha^{2}(i) M \Delta^{2}}{3 N^{2}}
\end{aligned}
$$

where we have used the independence assumptions and eqns. $(26,27)$. Continuing the recursion and using the fact that $\sum_{i>0} \alpha^{2}(i)<\infty$, we have

$$
\mathbb{E}\left[x_{\text {avg }}^{2}(i)\right] \leq x_{\text {avg }}^{2}(0)+\frac{2 M \Delta^{2}}{3 N^{2}} \sum_{j \geq 0} \alpha^{2}(j), \forall i
$$

We note that $\left\{x_{\text {avg }}(i)\right\}_{i \geq 0}$ is an $\mathcal{L}_{2}$-bounded martingale and hence converges a.s. and in $\mathcal{L}_{2}$ to a finite random variable $\theta$. In other words, there exists a finite random variable $\theta$, such that,

$$
\mathbb{P}\left[\lim _{i \rightarrow \infty} x_{\text {avg }}(i)=\theta\right]=1, \quad \lim _{i \rightarrow \infty} \mathbb{E}\left[x_{\text {avg }}(i)-\theta\right]^{2}=0
$$

The theorem then follows from eqns. $(24,32)$.

\section{MeAn-SQuared ERRor And Convergence Rate}

Section III shows that asymptotically the states of the sensors reach consensus and, in fact, converge a.s. to a

${ }^{3}$ A filtration, $\mathcal{F}$, is a non-decreasing sequence of sigma algebras. A stochastic process, $\{\mathbf{z}(i)\}_{i \geq 0}$, is $\mathcal{F}$ adapted, if $\mathbf{z}(i)$ is $\mathcal{F}_{i}$ measurable for each $i$. An integrable process, $\{\mathbf{z}(i)\}_{i \geq 0}$, which is adapted to a filtration $\mathcal{F}$, is a martingale if

$$
\mathbb{E}\left[\mathbf{z}(i+1) \mid \mathcal{F}_{i}\right]=\mathbf{z}(i) \text { a.s. }
$$


finite random variable $\theta$. Viewing $\theta$ as an estimate of $r$, we characterize its statistical properties. We show that $\theta$ is an unbiased estimate of $r$, which is a desirable property. To this end, we note that from eqn. (25) it follows

$$
\mathbb{E}\left[x_{\text {avg }}(i)\right]=r, \forall i \geq 0
$$

Since, the sequence $\left\{x_{\text {avg }}(i)\right\}_{i \geq 0}$ converges to $\theta$ in $\mathcal{L}_{2}$, it converges also in $\mathcal{L}_{1}$, and we have

$$
\begin{aligned}
\mathbb{E}[\theta] & =\lim _{i \rightarrow \infty} \mathbb{E}\left[x_{\mathrm{avg}}(i)\right] \\
& =r
\end{aligned}
$$

Thus, $\theta$ is an unbiased estimate of the desired average $r$. To compute the m.s.e. $\zeta$ (see eqn. (11)), we note that the sequence of non-negative functions $\left(x_{\mathrm{avg}}(i)-r\right)^{2}$ converges a.s. to $(\theta-r)^{2}$. Hence, by Fatou's lemma,

$$
\mathbb{E}[\theta-r]^{2} \leq \liminf _{i \rightarrow \infty} \mathbb{E}\left[x_{\mathrm{avg}}(i)-r\right]^{2}
$$

Using exactly similar manipulations, as used in the derivation of eqn. (31), it can be shown that

$$
\mathbb{E}\left[x_{\mathrm{avg}}(i)-r\right]^{2} \leq \frac{2 M \Delta^{2}}{3 N^{2}} \sum_{j \geq 0} \alpha^{2}(j), \forall i
$$

Combining eqns. $(35,36)$ it follows that

$$
\zeta \leq \frac{2 M \Delta^{2}}{3 N^{2}} \sum_{j \geq 0} \alpha^{2}(j)
$$

which gives an explicit upper bound on the m.s.e. We comment on this upper bound. We note that $\zeta$ increases with increasing $\Delta$. This is intuitive, because a coarser quantization will typically lead to larger m.s.e. We now point out another very interesting feature of the QC algorithm. Since, $\sum_{j \geq 0} \alpha^{2}(j)<$ $\infty$, we note that the m.s.e. $\zeta$ can be made as small as possible, by properly rescaling the weight sequence $\{\alpha(j)\}_{j \geq 0}$ by a constant. This means that the QC algorithm can be tuned to make $\zeta$ as small as desired. But, this leads to an interesting trade-off between $\zeta$ and the convergence rate of the algorithm. ${ }^{4}$ Thus, an attempt to increase the accuracy of the algorithm will lead to a slower convergence rate. This trade-off is studied formally in [2] and we omit it here due to lack of space.

\section{Numerical Studies}

We present a numerical study in this section. We consider $N=100$ sensors. The communication network is taken to be a Erdös-Rényi random graph with $M=5 N$ edges (the edges are chosen uniformly randomly from the set of all possible $N(N-1) / 2$ edges.) For half of the sensors, the initial data is sampled independently from a normal distribution with mean 50 and variance 10, while for the other half from a normal distribution with mean 70 and variance 10 . The quantization step-size is taken to be $\Delta=5$ and the weight sequence, as $\alpha(i)=1 / 5 i$. The blue line (with stars) in Fig. 1 denotes the path followed by the state of a randomly chosen node as the QC algorithm progressed, while the red line (solid) denotes

\footnotetext{
${ }^{4}$ By convergence rate, we mean here, informally, the rate at which the state vector sequence $\{\mathbf{x}(i)\}_{i \geq 0}$ converges to $\theta \mathbf{1}$.
}

the actual average $r$. Clearly, the plot verifies our theoretical results.

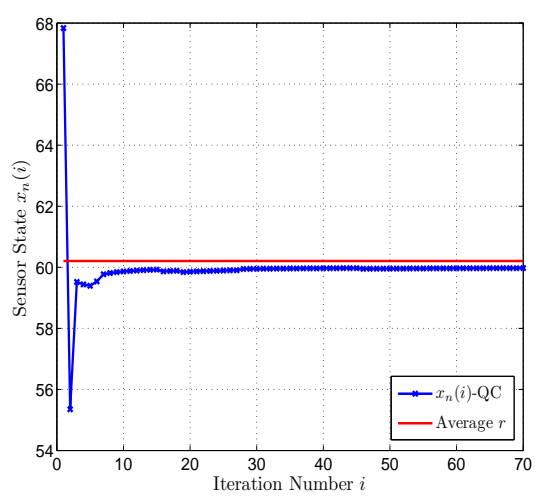

Fig. 1. QC algorithm on a network of $N=100$ sensors.

\section{Conclusion And Generalizations}

In this paper, we consider a distributed QC algorithm for average consensus when sensors exchange quantized state information. We show that, if the network is connected, the sensors asymptotically reach consensus and converge to a finite random variable $\theta$. We note that $\theta$ is an unbiased estimate of the desired average $r$, and also the mean-squared error, $\zeta$, between $\theta$ and $r$ can be made arbitrarily small, by rescaling the link weight sequence $\{\alpha(i)\}_{i \geq 0}$. We also point out the tradeoff between accuracy (smaller $\zeta$ ) and the convergence rate of the QC algorithm. Finally, we note that the QC algorithm can be extended to a wide range of situations involving imperfect inter-sensor communication. Examples include randomly failing communication links, additive channel noise, data dependent noise, etc. For example, in [2], we show that, if simultaneously we have additive channel noise and random Bernoulli link failures, a distributed algorithm can be designed, such that the sensors reach arbitrarily close to the desired average value, if $\lambda_{2}(\mathbb{E}[L])>0$ (note that the Laplacians are now random.)

\section{REFERENCES}

[1] R. Olfati-Saber and R. M. Murray, "Consensus problems in networks of agents with switching topology and time-delays," IEEE Trans. Automat. Contr., vol. 49, no. 9, pp. 1520-1533, Sept. 2004.

[2] S. Kar and J. M. F. Moura, "Distributed consensus algorithms in sensor networks with imperfect communication: Link failures, channel noise, quantized transmission," To be submitted.

[3] A. Kashyap, T. Basar, and R. Srikant, "Quantized consensus," Automatica, vol. 43, pp. 1192-1203, July 2007.

[4] T. C. Aysal, M. Coates, and M. Rabbat, "Distributed average consensus using probabilistic quantization," Madison, Wisconsin, USA, August 2007, pp. 640-644.

[5] F. R. K. Chung, Spectral Graph Theory. Providence, RI : American Mathematical Society, 1997.

[6] L. Schuchman, "Dither signals and their effect on quantization noise," IEEE Trans. Commun. Technol., vol. COMM-12, pp. 162-165, December 1964.

[7] M. Nevel'son and R. Has'minskii, Stochastic Approximation and Recursive Estimation. Providence, Rhode Island: American Mathematical Society, 1973. 